Supporting Information for

\title{
Ultrafast Dopant-Induced Exciton Auger-like Recombination in Mn-Doped Perovskite Nanocrystals
}

\author{
Shiping Wang, ${ }^{+, \neq}$Jing Leng, ${ }^{,+}+$Yanfeng Yin, ${ }^{\dagger}$ Junxue Liu, ${ }^{+}$Kaifeng Wu, ${ }^{+}$Shengye Jin ${ }^{*}+$ \\ ${ }^{+}$State Key Laboratory of Molecular Reaction Dynamics and Dynamics Research Center for Energy and \\ Environmental Materials, Dalian Institute of Chemical Physics, Chinese Academy of Sciences, Dalian \\ 116023, China \\ *University of Chinese Academy of Sciences, Beijing 100049, China
}

\section{Synthesis of $\mathrm{CsPbCl}_{3}$ and $\mathrm{Mn}$-doped $\mathrm{CsPbCl}_{3} \mathrm{NCs}$}

Undoped and $\mathrm{Mn}$-doped $\mathrm{CsPCl}_{3} \mathrm{NCs}$ were synthesized by using a modified hot-injection method. Cesium-oleate was prepared as follows. $\mathrm{Cs}_{2} \mathrm{CO}_{3}(0.407 \mathrm{~g})$, oleic acid $(\mathrm{OA}, 1.5 \mathrm{~mL})$ and octadecylene (ODE, $20 \mathrm{~mL}$ ) were added into a $50 \mathrm{~mL}$ two-neck flask, degassed and heated under vacuum for $1 \mathrm{~h}$ at $130^{\circ} \mathrm{C}$. When the mixture changed from turbid to clear, the Cs-oleate solution was cooled to $100^{\circ} \mathrm{C}$. To synthesize of the undoped $\mathrm{CsPbCl}_{3}$ samples, $\mathrm{PbCl}_{2}(0.209 \mathrm{~g})$, ODE (20mL), OA (2mL), and oleylamine (OLA, 2mL) were loaded into a $100 \mathrm{~mL}$ three-neck flask. For the synthesis of the Mn-doped $\mathrm{NCs}, \mathrm{PbCl}_{2}(0.209 \mathrm{~g})$, anhydrous $\mathrm{MnCl}_{2}(0.0946 \mathrm{~g})$, ODE (20mL), OA $(2 \mathrm{~mL})$, OLA $(2 \mathrm{~mL})$ were loaded into a $100 \mathrm{~mL}$ three-neck flask. Both mixed solutions were degassed and heated under vacuum for $1 \mathrm{~h}$ at $120^{\circ} \mathrm{C}$. Then, the temperature was raised to $180^{\circ} \mathrm{C}$ rapidly, and $1.6 \mathrm{~mL}$ of Cs-oleate solution mentioned above was swiftly injected. After 5-10 seconds, the reaction mixture was cooled down to room temperature by an ice-water bath. Then, the mixture was separated by centrifuging for $15 \mathrm{~min}$ at $5000 \mathrm{rpm}$. The precipitate was re-dispersed in $5 \mathrm{~mL}$ of hexane and centrifuged for $10 \mathrm{~min}$ at 5000rpm to obtain supernatant for further measurements.

\section{Synthesis of Mn-doped $\mathrm{CsPb}(\mathrm{Cl} / \mathrm{Br})_{3} \mathrm{NCs}$}


The synthesis of $\mathrm{Mn}$-doped $\mathrm{CsPb}(\mathrm{Cl} / \mathrm{Br})_{3} \mathrm{NCs}$ is almost the same as that of $\mathrm{CsPbCl}_{3} \mathrm{NCs}$, excluding a slight chemical feeding change. To synthesize Mn-doped mixed halogen perovskite $\left(\mathrm{CsPb}(\mathrm{Cl} / \mathrm{Br})_{3}\right) \mathrm{NCs}, \mathrm{PbCl}_{2}(0.084 \mathrm{~g}), \mathrm{PbBr}_{2}(0.041 \mathrm{~g})$, anhydrous $\mathrm{MnCl}_{2}(0.047 \mathrm{~g})$, ODE $(20 \mathrm{~mL})$, OA $(2 \mathrm{~mL})$, OLA $(2 \mathrm{~mL})$ were loaded into a $100 \mathrm{~mL}$ three-neck flask. For the degassing, injection, and purification procedures, we repeated the above operation procedure like the synthesis of $\mathrm{CsPbCl}_{3} \mathrm{NCs}$. By using inductive-coupled plasma mass spectrometry (ICP-MS), the doping ratio of $\mathrm{Br}^{-}(\mathrm{Br} / \mathrm{Cl})$ in the $\mathrm{NCs}$ is estimated to be $\sim 3 \%$ and the doping ratio of $\mathrm{Mn}^{2+}$ is estimated to be $\sim 2.5 \%$ relative to $\mathrm{Pb}^{2+}$. The steady absorption and PL spectra of as-prepared $\mathrm{Mn}$-doped $\mathrm{CsPb}(\mathrm{Cl} / \mathrm{Br})_{3} \mathrm{NCs}$ were shown in Figure S8.

\section{Structural and spectral characterization of NCs}

Transmission electron microscopy (TEM) and high resolution transmission electron microscopy (HRTEM) of doped and undoped $\mathrm{CsPbCl}_{3}$ nanocrystals were measured by a JEM-2100. The UV-vis absorption spectra of both samples were obtained using a Cary 60 UV-vis spectrometer of Agilent. The PL decay curve of $\mathrm{Mn}^{2+}$ emission was obtained on a FLS920 of Edinburgh Instruments. The XRD spectra were obtained on an X'pert Pro-1. The PL spectra were obtained using an Ocean Optics spectrometer. The host exciton PL lifetime of doped and undoped $\mathrm{CsPbCl}_{3}$ QDs were obtained on a FluoroMax-4 of Horiba Instruments.

\section{PL up-conversion experiment}

The femtosecond PL up-conversion (u-PL) experiments were performed on a Chimera spectrometer (Light conversion) using a Pharos laser (1030 nm,100 kHz, $230 \mathrm{fs}$ pulse-duration; Light conversion) as the excitation and gate sources. One part of the Pharos output was used to pump an optical parametric amplifier (OPA; TOPAS) to generate the wavelength-tunable excitation pulses, while the other was used as the gate pulse. The light emitted by the sample was collected by lens and focused into a BBO crystal together with the gate pulse to generate the up-converted signal via sum frequency generation (SFG). The signal photons were focused onto the entrance slit of a monochromator and detected by the spectrometer. The time-resolved decay curve was obtained by delaying the gate pulse with 
respect to the pump pulse using a mechanical delay stage. The samples were placed in $2 \mathrm{~mm}$ quartz cuvettes and were vigorously stirred in all the measurements in order to minimize photocharging or damaging of the samples.

\section{TA experiment}

Pump-probe TA experiment. The femtosecond transient absorption setup is based on a regenerative amplified Ti:sapphire laser from Coherent corporation (800 nm, $35 \mathrm{fs}, 6$ $\mathrm{mJ} /$ pulse, and $1 \mathrm{kHz}$ repetition rate), nonlinear frequency mixing techniques and a transient absorption spectrometer (TA100) from Time-tech Spectra LLC. Briefly, the $800 \mathrm{~nm}$ output pulse from the regenerative amplifier was split in two parts with a $50 \%$ beam splitter. The transmitted part was used to pump a TOPAS Optical Parametric Amplifier (OPA) which generated a wavelength-tunable laser pulse from $250 \mathrm{~nm}$ to $2.5 \mu \mathrm{m}$ as pump beam. The reflected $800 \mathrm{~nm}$ beam was split again into two parts. One part with less than $10 \%$ was attenuated with a neutral density filter and focused into a $2 \mathrm{~mm}$ thick sapphire or $\mathrm{CaF}_{2}$ window to generate a white light continuum (WLC) used for probe beam. The probe beam was focused with an Al parabolic reflector onto the sample. After the sample, the probe beam was collimated and then focused into a fiber-coupled spectrometer with CMOS sensors and detected at a frequency of $1 \mathrm{KHz}$. The intensity of the pump pulse used in the experiment was controlled by a variable neutral-density filter wheel. The delay between the pump and probe pulses was controlled by a motorized delay stage. The pump pulses were chopped by a synchronized chopper at $500 \mathrm{~Hz}$ and the absorbance change was calculated with two adjacent probe pulses (pump-blocked and pump-unblocked). The samples were placed in $2 \mathrm{~mm}$ cuvettes and vigorously stirred in all the measurements. All TA experiments were performed under ambient conditions.

Pump-pump-probe TA experiment. The pump-pump-probe TA experiments were performed by adding another pump pulse into the regular TA set-ups. Specifically, the $350 \mathrm{~nm}$ pump beam used above was split into two beams. The delay time between these two pump pulses was created by delaying one of them using a longer path length. In our experiment, the 
delay was fixed respectively at $\sim 8 \mathrm{~ns}$ for $\mathrm{Mn}$-doped $\mathrm{CsPbCl}_{3} \mathrm{NCs}$ and $\sim 20 \mathrm{~ns}$ for Mn-doped $\mathrm{CsPb}(\mathrm{Cl} / \mathrm{Br})_{3} \mathrm{NCs}$. The delay between the second pump pulse and the probe pulse was controlled by a motorized delay stage. These two beams were overlapped with the probe beam on the sample. The first pump beam is always on while the second one is chopped at $500 \mathrm{~Hz}$. The intensities of both pump beams were controlled by neutral-density filters.

\section{Kinetics fitting models for excited Mn-induced Auger-like recombination}

As mentioned in main text, in spite of the relatively high excitation intensity $\left(1.75 \mathrm{~mJ} / \mathrm{cm}^{2}\right.$, not enough to saturation) of the first pump pulse used in the ppp-TA experiment, there still exists a subset of doped NCs that are unexcited by the first pulse but excited by the second one in the ppp-TA measurement. In this case, the ppp-TA is only measuring the NCs excited by only one pulse, which is essentially the same as regular pp-TA. Thus, by normalizing ppp-TA and pp-TA kinetics to their slowly decaying component (as in Figure 4a) and performing a subtraction between them, we can exclude the contribution from pp-TA kinetics and obtain pure decay kinetics for the doped NCs in the presence of the excited $\mathrm{Mn}^{2+}$.

In Mn-doped $\mathrm{CsPbCl}_{3} \mathrm{NCs}$ with co-existing host exciton and excited $\mathrm{Mn}^{2+}$, there are two main decay channels for the host excitons (see Figure S9): 1) the holes are first trapped by defects and then, together with the electrons left at the band edge, undergo the Auger-like recombination induced by the excited $\mathrm{Mn}^{2+} ; 2$ ) host excitons in doped NCs directly undergo Auger-like recombination induced by the excited $\mathrm{Mn}^{2+}$. Assuming that the two kinds of Auger-like recombination induced by the excited $\mathrm{Mn}^{2+}$ have same time constant, then the XB kinetics in the doped NCs with co-existing host exciton and excited-state $\mathrm{Mn}^{2+}$, which also represents the difference kinetics shown in Figure $4 \mathrm{~b}$, can be described by the following three-exponential function:

$$
\Delta X B(t)=-a_{1} \exp \left(-t / \tau_{1}\right)-a_{2} \exp \left(-t / \tau_{2}\right)-a_{3} \exp \left(-t / \tau_{A u g}\right)
$$

where $\tau_{1}$ and $\tau_{2}$ represent the time constants of hole trapping in $\mathrm{Mn}$-doped $\mathrm{CsPbCl}_{3} \mathrm{NCs}, \tau_{\mathrm{Aug}}$ represents the dopant-induced Auger-like recombination time. When fitting the kinetics in Figure $4 \mathrm{~b}$ in terms of Eq.(S1), we fixed these two time constants of hole trapping obtained via 
fitting ultrafast PL kinetics, and simply used $\tau_{\mathrm{Aug}}, a_{1}, a_{2}$ and $a_{3}$ as free fitting parameters (see Table S2). It must be noted that, for $\mathrm{Mn}$-doped $\mathrm{CsPbCl}_{3} \mathrm{NCs}$, since the hole trapping rate $\left(\tau_{1}=2.1 \mathrm{ps}\right)$, which dominates its PL kinetics (Table S1), is much faster than the measured excited $\mathrm{Mn}^{2+}$ induced Auger-like recombination rate $\left(\tau_{\text {Aug }}=12.1 \mathrm{ps}\right)$, the main decay channel for the host excitons in the NCs with co-existing excited $\mathrm{Mn}^{2+}$ should be the first case as mentioned above. Thus, the dopant-induced Auger-like recombination rate $\left(\tau_{\mathrm{Aug}}=12.1 \mathrm{ps}\right)$ measured here should also be mainly related to the first Auger-like recombination process, i.e., Auger-like recombination (1) in Figure S9b. 


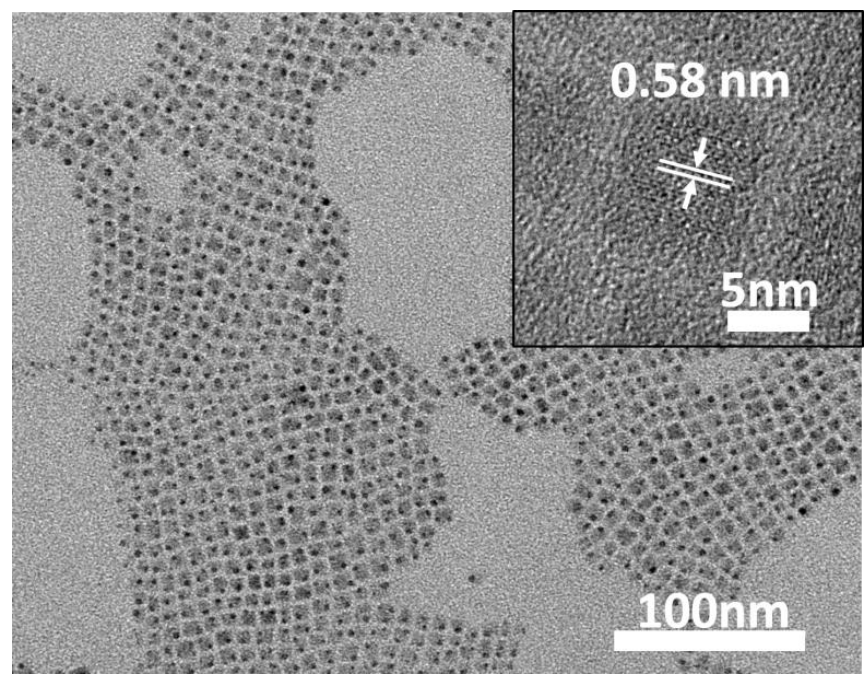

Figure S1. TEM and (inset) HRTEM images of undoped $\mathrm{CsPbl}_{3} \mathrm{NCs}$.

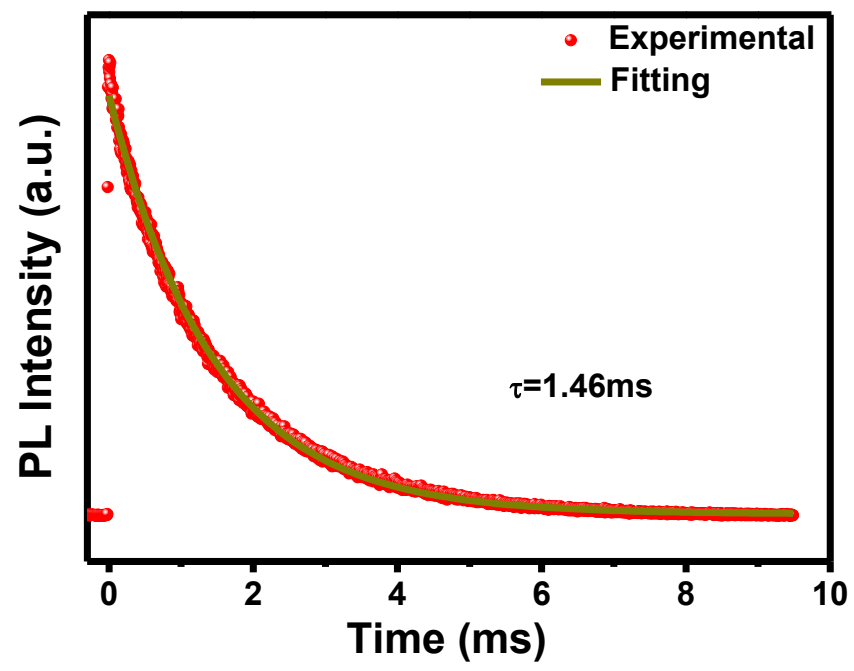

Figure S2. PL decay trace of $\mathrm{Mn}^{2+}$ emission in $\mathrm{Mn}$-doped $\mathrm{CsPbCl}_{3} \mathrm{NCs}$. 


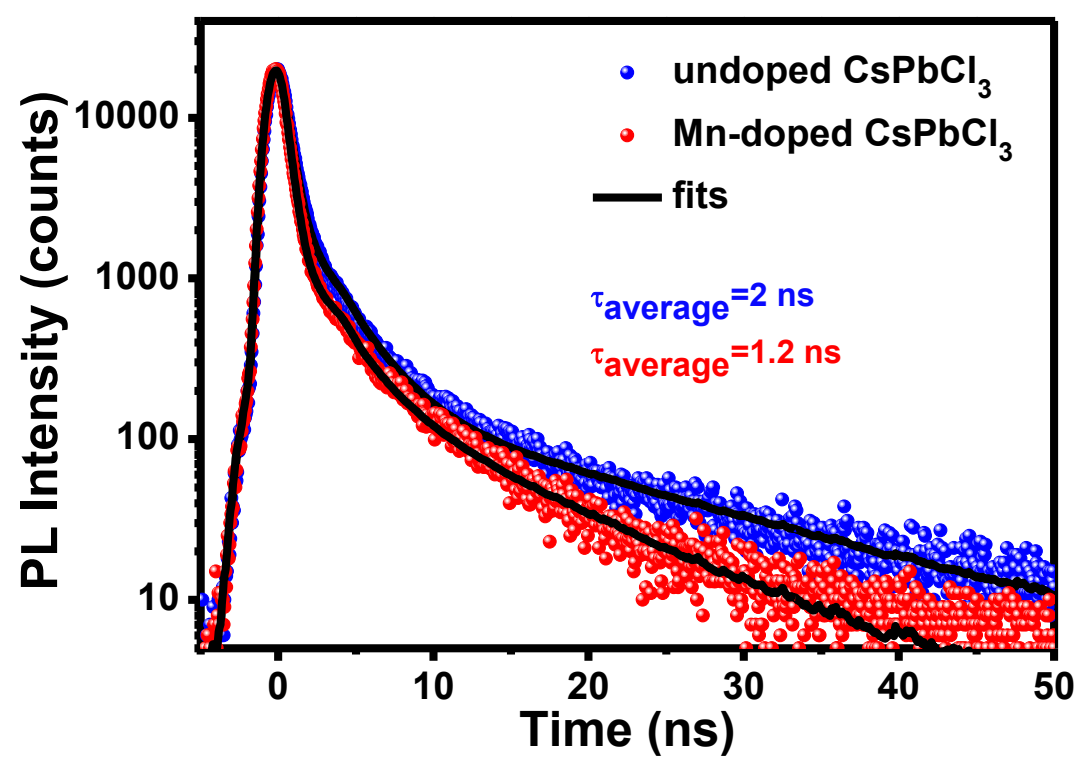

Figure S3. PL decay traces of host excitons for undoped and $\mathrm{Mn}$-doped $\mathrm{CsPbCl}_{3} \mathrm{NCs}$ measured respectively by TCSPC technique. 

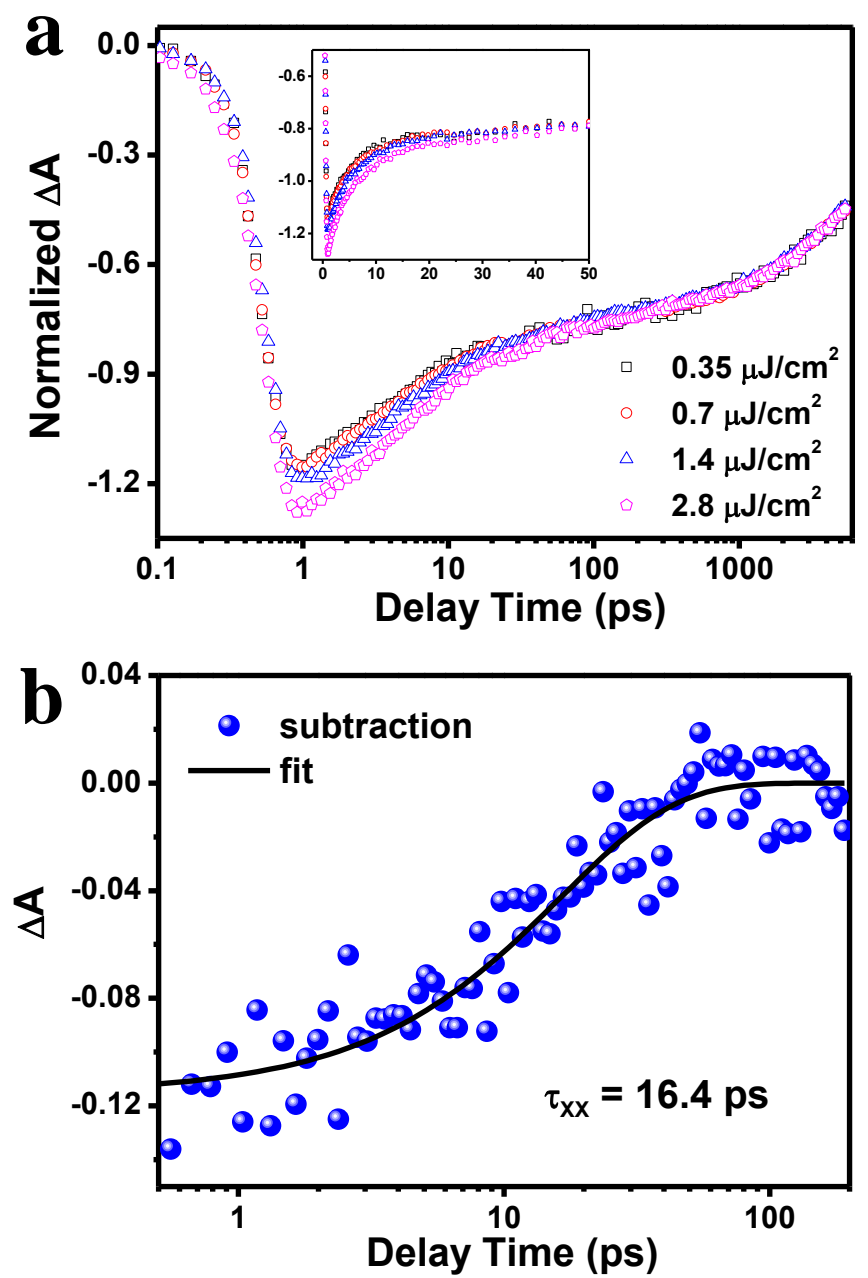

Figure S4. (a) TA kinetics of undoped $\mathrm{CsPbCl}_{3} \mathrm{NCs}$ normalized to the long lived tail under different excitation intensities. (b) TA kinetics of bi-exciton recombination obtained by performing a subtraction between the kinetic traces (normalized to the long lived tail) of $\mathrm{CsPbCl}_{3}$ under 2.8 and $0.35 \mu \mathrm{J} / \mathrm{cm}^{2}$ excitation intensities. The black solid line is a single-exponential fit to the kinetics, yielding a bi-exciton lifetime of $\tau_{\mathrm{XX}}=16.4 \pm 1.3 \mathrm{ps}$. 


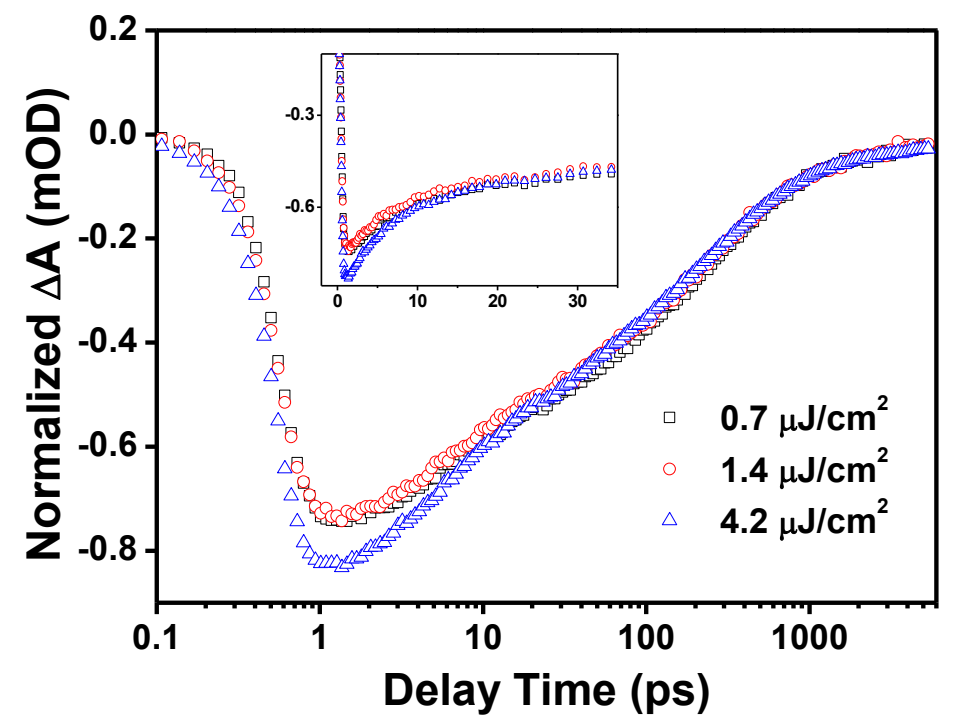

Figure S5. TA kinetics of $\mathrm{Mn}$-doped $\mathrm{CsPbCl}_{3} \mathrm{NCs}$ normalized to the long lived tail under different excitation intensities.

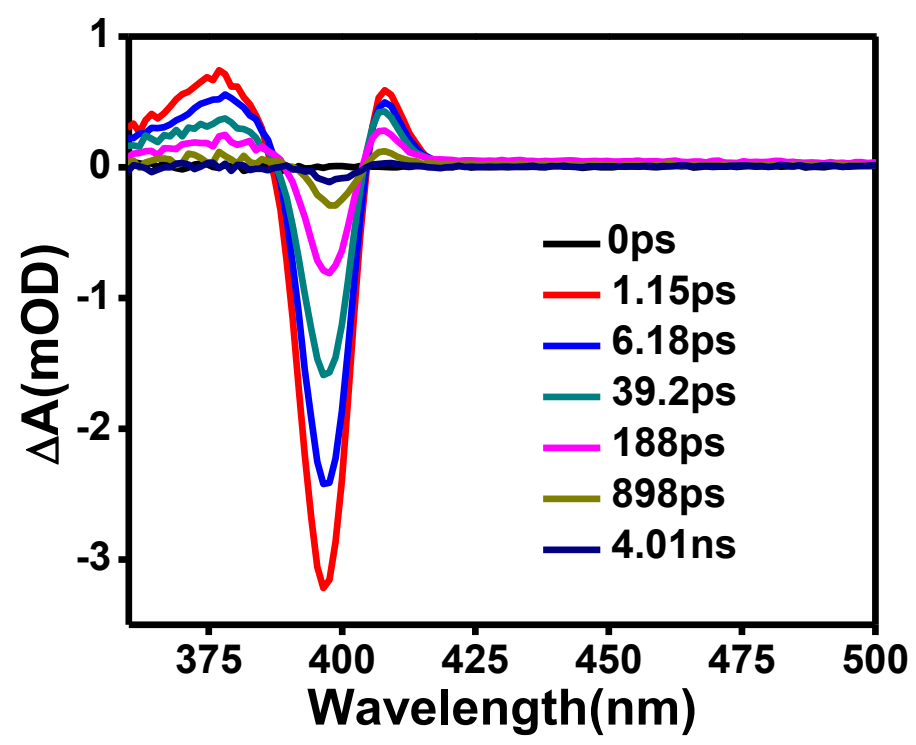

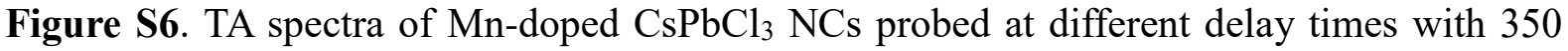
$\mathrm{nm}$ excitation by using ppp-TA method. In this experiment, the first pulse energy is 1.75 $\mathrm{mJ} / \mathrm{cm}^{2}$, while the second pulse energy is $0.7 \mu \mathrm{J} / \mathrm{cm}^{2}$. 


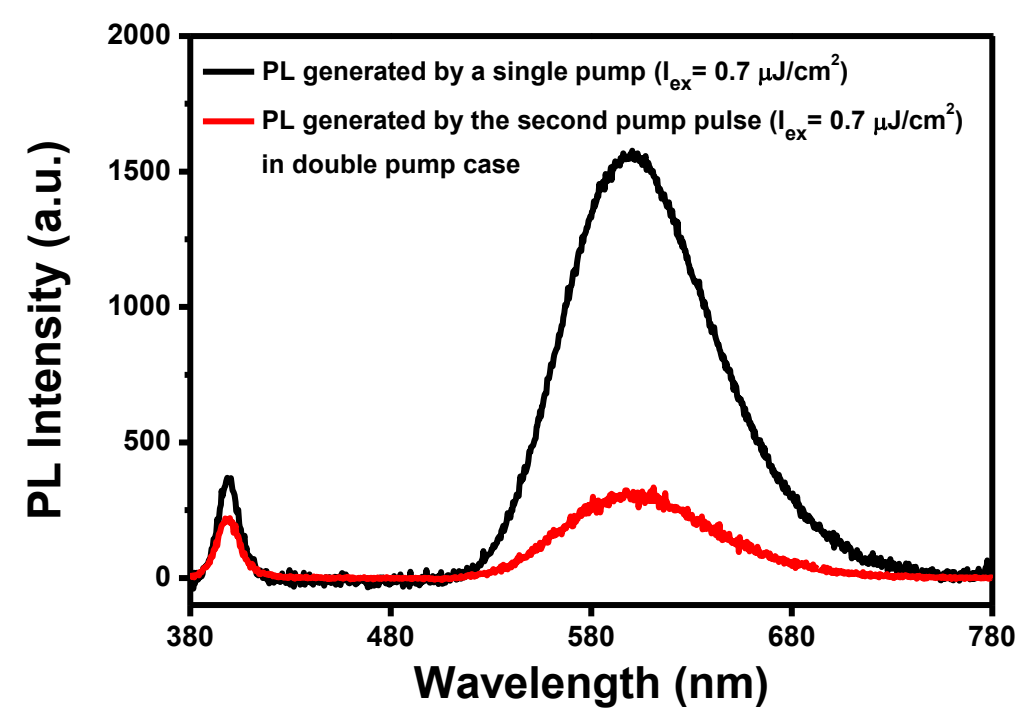

Figure S7. PL spectrum of Mn-doped $\mathrm{CsPbl}_{3} \mathrm{NCs}$ generated by a single pump excitation (as in pp-TA measurement) (black line) and the PL spectrum of the same NCs generated by the second pump pulse in a double pump condition (as in ppp-TA measurement) (red line). For the double pump case, the first excitation pulse is $1.75 \mathrm{~mJ} / \mathrm{cm}^{2}$ with $1000-\mathrm{Hz}$ repetition rate, while the second excitation pulse is $0.7 \mu \mathrm{J} / \mathrm{cm}^{2}$ with $500-\mathrm{Hz}$ repetition rate and the delay between the two pump pulses for double pump case is also fixed at $\sim 8 \mathrm{~ns}$. For the simple pump PL, the pump intensity is $0.7 \mu \mathrm{J} / \mathrm{cm}^{2}$, which is the same as the second pump pulse in double pump case. 

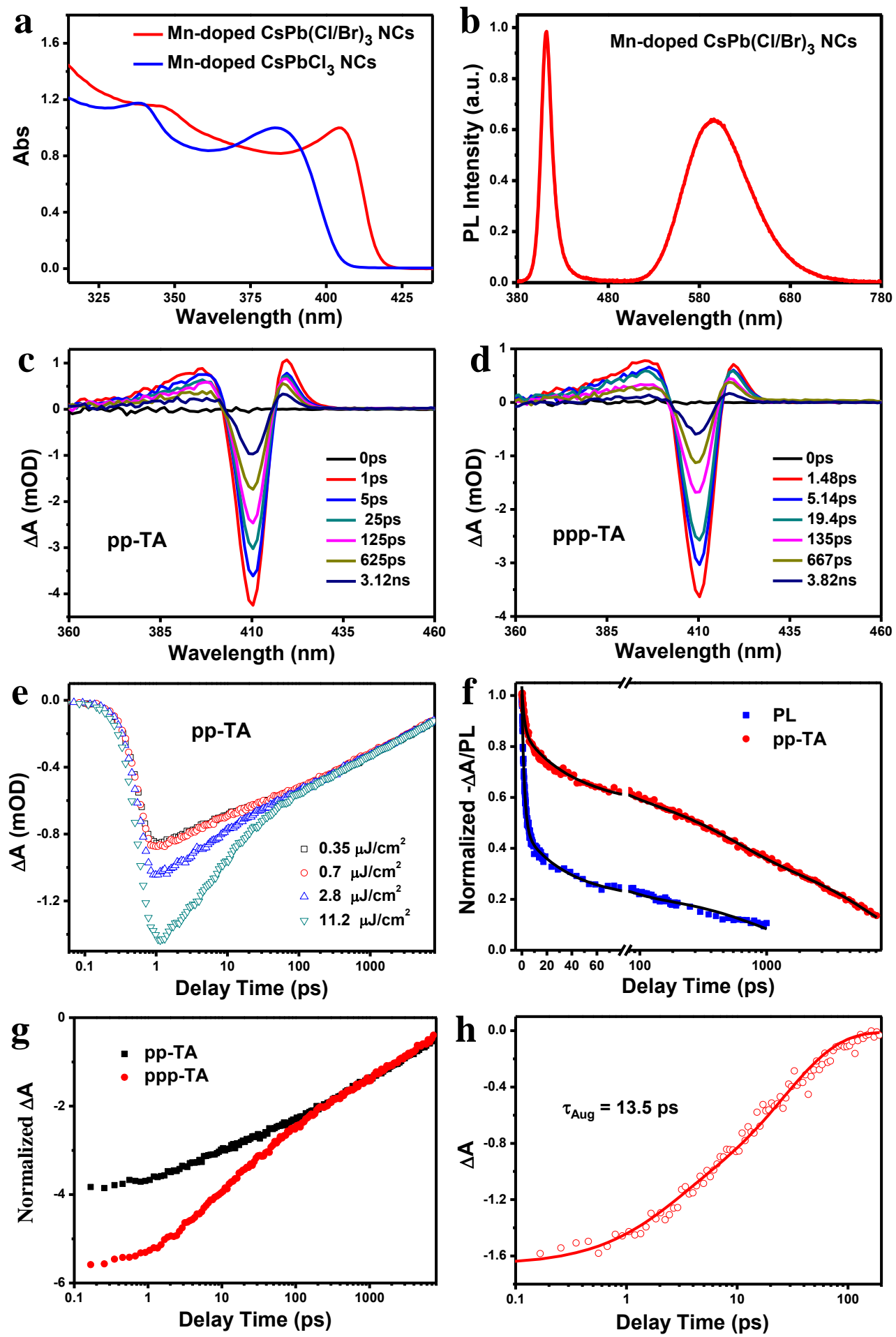

Figure S8. The spectral characterization and photoinduced carrier dynamics of Mn-doped $\mathrm{CsPb}(\mathrm{Cl} / \mathrm{Br})_{3}$ NCs. (a) UV-vis absorption spectra and (b) PL spectra of Mn-doped $\mathrm{CsPb}(\mathrm{Cl} / \mathrm{Br})_{3} \mathrm{NCs}$. (c) pp-TA and (d) ppp-TA spectra of $\mathrm{Mn}$-doped $\mathrm{CsPb}(\mathrm{Cl} / \mathrm{Br})_{3} \mathrm{NCs}$ at different delay times under excitation at $350 \mathrm{~nm}$ with the excitation energy same as $\mathrm{Mn}$-doped $\mathrm{CsPbCl}_{3} \mathrm{NCs}$. Considering the longer carrier lifetime in $\mathrm{Mn}$-doped $\mathrm{CsPb}(\mathrm{Cl} / \mathrm{Br})_{3}$ NCs, the delay between the two pump pulses in this ppp-TA experiment was fixed at $\sim 20 \mathrm{~ns}$. 
(e) TA kinetics of $\mathrm{Mn}$-doped $\mathrm{CsPb}(\mathrm{Cl} / \mathrm{Br})_{3} \mathrm{NCs}$ normalized to the long-lived tail under different excitation intensities. (f) Normalized XB recovery kinetics and ultrafast exciton PL decay kinetics in Mn-doped $\mathrm{CsPb}(\mathrm{Cl} / \mathrm{Br})_{3}$ NCs. Solid lines are fits to these kinetics by a multi-exponential function with fitting parameters listed in Table S3. (g) The comparison of XB kinetics of $\mathrm{Mn}$-doped $\mathrm{CsPb}(\mathrm{Cl} / \mathrm{Br})_{3} \mathrm{NCs}$ measured by using pp-TA and ppp-TA, showing the faster decay in ppp-TA kinetics and thus demonstrating the existence of the fast Auger-like recombination in the NCs. The kinetics are normalized to their slowly decaying components. (h) Kinetics trace obtained by performing a subtraction between pp-TA and ppp-TA kinetic in panel $\mathrm{g}$. The red solid line is a fit of the kinetics according to Eq. (S1) in SI, yielding $\tau_{\mathrm{Aug}}=13.5 \pm 0.8 \mathrm{ps}$.
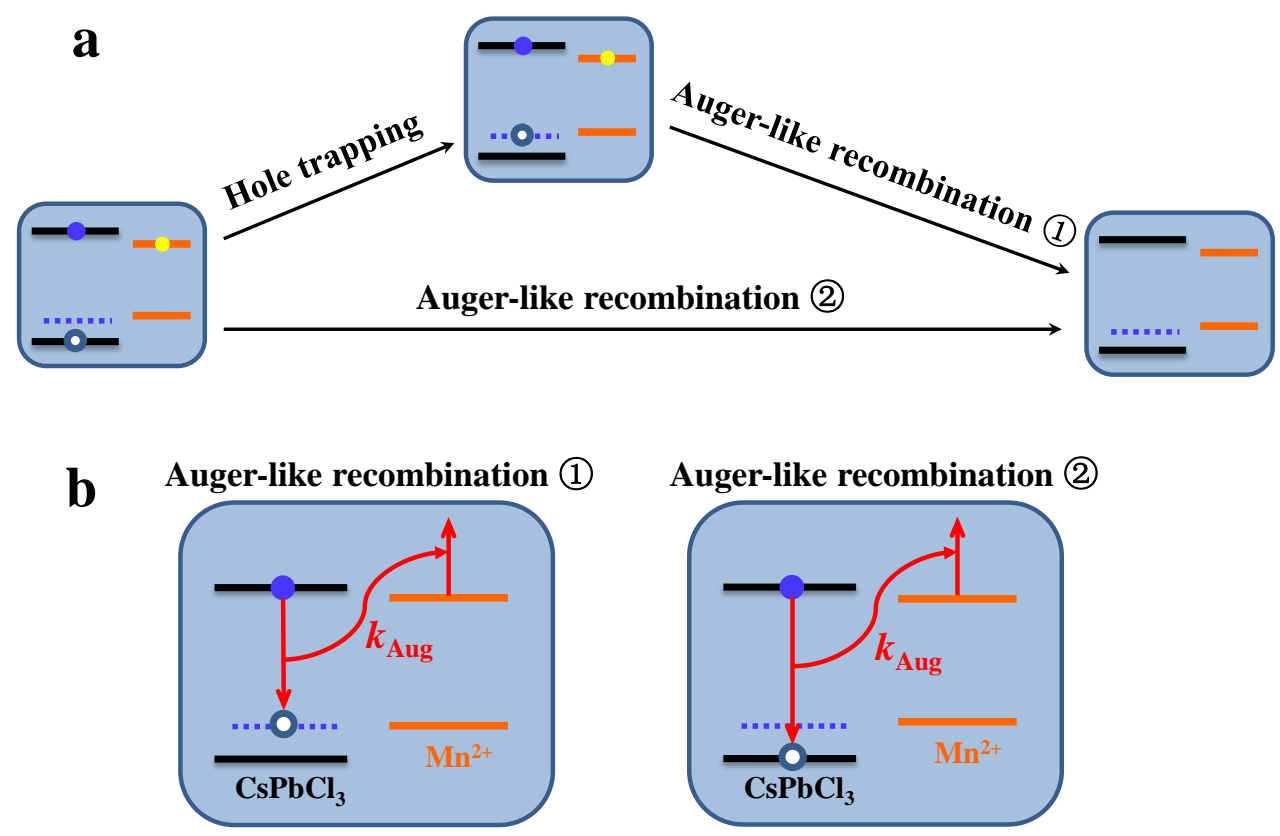

Figure S9. (a) Scheme for the decay channel of host excitons in $\mathrm{Mn}$-doped $\mathrm{CsPbCl}_{3} \mathrm{NCs}$ with co-existing excited-state $\mathrm{Mn}^{2+}$. (b) Scheme for the Auger-like recombination process induced by the excited $\mathrm{Mn}^{2+}$ (1) and (2)) in Mn-doped $\mathrm{CsPbCl}_{3} \mathrm{NCs}$. 
Table S1. Fitting parameters for normalized PL and TA kinetics shown in Figure 3c. The kinetics are fit by a multiple-exponential decay function $\left(y=-\Sigma_{\mathrm{i}} \mathrm{a}_{\mathrm{i}} \exp \left(-\mathrm{t} / \tau_{\mathrm{i}}\right)\right)$.

\begin{tabular}{|c|c|c|c|c|c|}
\hline Samples & & $\begin{array}{c}\tau_{1} / \mathbf{p s} \\
\left(\mathbf{a}_{1}\right) \\
\end{array}$ & $\begin{array}{c}\tau_{2} / \mathbf{p s} \\
\left(\mathbf{a}_{2}\right) \\
\end{array}$ & $\begin{array}{c}\tau_{3} / \mathbf{p s} \\
\left(\mathrm{a}_{3}\right)\end{array}$ & $\begin{array}{c}\tau_{4}\left(\tau_{\text {IET }}\right) / \mathrm{ps} \\
\left(\mathbf{a}_{4}\right)\end{array}$ \\
\hline \multirow{2}{*}{$\mathrm{CsPbCl}_{3}$} & PL & $\begin{array}{c}1.9 \pm 0.1 \\
(65.7 \pm 1.1 \%)\end{array}$ & $\begin{array}{c}26.8 \pm 2.2 \\
(22.1 \pm 0.8 \%)\end{array}$ & $\begin{array}{c}1314.7 \pm 143.8 \\
(12.2 \pm 0.5 \%)\end{array}$ & \\
\hline & TA & $\begin{array}{c}1.9 \text { (fixed) } \\
(18.6 \pm 0.5 \%)\end{array}$ & $\begin{array}{c}26.8 \text { (fixed) } \\
(17.6 \pm 0.3 \%) \\
\end{array}$ & $\begin{array}{c}9580.3 \pm 154.4 \\
(63.8 \pm 0.2 \%) \\
\end{array}$ & \\
\hline \multirow{2}{*}{$\begin{array}{c}\text { Mn-doped } \\
\mathrm{CsPbCl}_{3}\end{array}$} & $\mathbf{P L}$ & $\begin{array}{c}2.1 \pm 0.1 \\
(61.9 \pm 0.8 \%) \\
\end{array}$ & $\begin{array}{c}35.6 \pm 2.2 \\
(25.8 \pm 0.6 \%) \\
\end{array}$ & $\begin{array}{c}552.8 \pm 37.8 \\
(12.3 \pm 0.6 \%)\end{array}$ & \\
\hline & TA & $\begin{array}{c}2.1 \text { (fixed) } \\
(19.5 \pm 0.7 \%)\end{array}$ & $\begin{array}{c}35.6 \text { (fixed) } \\
(17.4 \pm 0.3 \%)\end{array}$ & $\begin{array}{c}9580.3 \text { (fixed) } \\
(8.2 \pm 0.4 \%)\end{array}$ & $\begin{array}{c}302.6 \pm 7.0 \\
(54.9 \pm 0.4 \%)\end{array}$ \\
\hline
\end{tabular}

Note: The IET efficiency can be approximately evaluated to be $\sim 87 \%$ in terms of $\eta=a_{4} /\left(a_{3}+a_{4}\right)$.

Table S2. Fitting parameters for the difference kinetics shown in Figure 4b. The kinetics are fit in terms of Eq.(S1).

\begin{tabular}{c|c|c|c}
\hline \hline Samples & $\begin{array}{c}\tau_{1} / \mathrm{ps} \\
\left(\mathrm{a}_{1}\right)\end{array}$ & $\begin{array}{c}\tau_{2} / \mathrm{ps} \\
\left(\mathrm{a}_{2}\right)\end{array}$ & $\begin{array}{c}\tau_{\mathrm{Aug}} / \mathrm{ps} \\
\left(\mathrm{a}_{3}\right)\end{array}$ \\
\hline $\begin{array}{c}\text { Mn-doped } \\
\mathrm{CsPbCl}_{3}\end{array}$ & 2.1 (fixed) & 35.6 (fixed) & $12.1 \pm 1.3$ \\
$(22.5 \pm 2.6 \%)$ & $(27.8 \pm 3.4 \%)$ & $(49.6 \pm 2.7 \%)$ \\
\hline \hline
\end{tabular}

Table S3. Fitting parameters for normalized PL and TA kinetics shown in Figure S8f. The kinetics are fit by a multiple-exponential decay function $\left(y=-\Sigma_{\mathrm{i}} \mathrm{a}_{\mathrm{i}} \exp \left(-\mathrm{t} / \tau_{\mathrm{i}}\right)\right)$.

\begin{tabular}{|c|c|c|c|c|c|}
\hline Samples & & $\begin{array}{c}\tau_{1} / \mathbf{p s} \\
\left(\mathbf{a}_{1}\right)\end{array}$ & $\begin{array}{c}\tau_{2} / \mathbf{p s} \\
\left(\mathbf{a}_{2}\right)\end{array}$ & $\begin{array}{c}\tau_{3} / \mathbf{p s} \\
\left(\mathbf{a}_{3}\right)\end{array}$ & $\begin{array}{c}\tau_{4}\left(\tau_{\text {IET }}\right) / \mathrm{ps} \\
\left(\mathbf{a}_{4}\right)\end{array}$ \\
\hline \multirow{2}{*}{$\begin{array}{c}\text { Mn-doped } \\
\mathrm{CsPb}(\mathrm{Cl} / \mathrm{Br})_{3}\end{array}$} & PL & $\begin{array}{c}2.0 \pm 0.1 \\
(53.3 \pm 0.7 \%)\end{array}$ & $\begin{array}{c}34.9 \pm 3.1 \\
(24.3 \pm 0.7 \%)\end{array}$ & $\begin{array}{c}1002.8 \pm 77.4 \\
(22.4 \pm 0.6 \%)\end{array}$ & \\
\hline & TA & $\begin{array}{c}2.0 \text { (fixed) } \\
(17.2 \pm 0.5 \%)\end{array}$ & $\begin{array}{l}34.9 \text { (fixed) } \\
(21.7 \pm 0.6 \%)\end{array}$ & $\begin{array}{c}6816.8 \pm 300.2 \\
(36.7 \pm 0.8 \%)\end{array}$ & $\begin{array}{c}466.5 \pm 30.6 \\
(24.4 \pm 0.8 \%)\end{array}$ \\
\hline
\end{tabular}

